\title{
A rare clinical association: Barth syndrome and cystic fibrosis
}

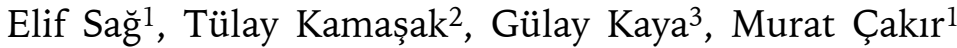 \\ Departments of ${ }^{1}$ Pediatric Gastroenterology Hepatology and Nutrition, ${ }^{2}$ Pediatric Neurology and ${ }^{3}$ Pediatric Intensive Care \\ Medicine, Karadeniz Technical University, Faculty of Medicine, Trabzon, Turkey. E-mail: muratcak@hotmail.com \\ Received: 26th August 2017, Revised: 29th December 2017, 3rd January 2018, Accepted: 5th January 2018
}

\begin{abstract}
SUMMARY: Sağ E, Kamaşak T, Kaya G, Çakır M. A rare clinical association: Barth syndrome and cystic fibrosis. Turk J Pediatr 2019; 61: 134-138.

Barth syndrome (BS) is a rare X-linked recessive metabolic disorder characterized by cardiomyopathy, hypotonia, neutropenia, growth retardation and 3-methylglutaconic aciduria type II. Cystic fibrosis is a common autosomal recessive genetic disorder in Caucasians. Herein, we reported a rare clinical association in an infant diagnosed based on clinical and genetic analysis. A six-month old boy admitted with chronic steatorrhea. The diagnosis of cystic fibrosis was made after clinical and laboratory examinations. Fifteen days later, the patient was presented with restlessness and moaning. He had hypoglycemia and lactic acidosis. The patient died three hours after the admission. Pedigree analysis revealed similar sudden infant deaths in close relatives. Postmortem genetic analysis revealed the diagnosis of Barth syndrome. This is the first case of the association of Barth syndrome with cystic fibrosis. Our case reinforces the importance of pedigree analysis and postmortem examinations.
\end{abstract}

Key words: Barth syndrome, cystic fibrosis, pedigree.

Barth syndrome (BS) is a rare X-linked recessive metabolic disorder characterized by cardiomyopathy, hypotonia, neutropenia, growth retardation and 3-methylglutaconic aciduria (3-MGA) type II. ${ }^{1}$ The disease has been shown to be associated with decreased tafazzin synthesis and concomitant reduction in cardiolipin (CL) levels at the mitochondrial level as a result of the mutation in the TAZ gene. ${ }^{2} \mathrm{CL}$ plays crucial roles in various cellular processes such as apoptosis, iron metabolism, mitochondrial electron transport, autophagy, cell membrane formation, and intercellular communication. ${ }^{2}$ As a consequence of mutation in the TAZ gene, CL level is decreased whereas the level of monolysocardiolipin is relatively increased, leading to mitochondrial dysfunction, the formation of reactive oxygen radicals and cell death. ${ }^{2}$

Cystic fibrosis (CF) is a common autosomal recessive genetic disorder in Caucasians. The $\mathrm{CF}$ gene is located in the q22-31 region of the long arm of $7^{\text {th }}$ chromosome and encodes the cystic fibrosis transmembrane regulator (CFTR) protein, which is found in numerous cell types localized in airway epithelium, pancreas, liver and sweat glands. Mutations in this gene affect the transport of chloride across the cell membrane, and up to now, over 1,900 mutations have been described that lead to this disease. The most important complications are related to lungs and account for $90 \%$ of the mortality. In addition to pulmonary involvement, steatorrhea related to pancreatic involvement results in growth retardation in the majority of cases. ${ }^{3}$

In this article, we report a patient who initially presented to our outpatient clinic with the complaint of fatty diarrhea but died during the follow-up period. The patient was diagnosed with BS as a result of detailed medical history, pedigree, and genetic analysis. Further analysis regarding steatorrhea also provided $\mathrm{CF}$ diagnosis. We considered it reasonable to report this case to share our experience about the process related to the extremely rare coexistence of these two genetic diseases.

\section{Case Report}

A six-month-old male infant was brought with chronic diarrhea and nutritional problems, 


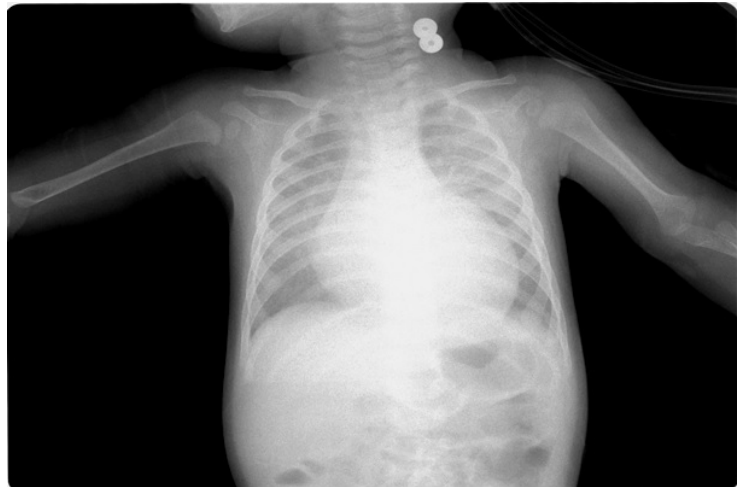

Fig. 1. Cardiomegaly detected by telecardiography.

which started three weeks prior to admission. The patient was the second child of the mother, who did not have any abortion, stillbirth or death child history. He was fed with breast milk for the first four months, and standard formulamilk was added to the nutrition program of the patient since breast milk was insufficient. The frequency of diarrhea was 4-5 times a day, it had a foul odor, was bright in color and was not bloody. The patient was born by cesarean section with a birth weight of 3,900 grams $\left(50^{\text {th }}\right.$ $-75^{\text {th }} \mathrm{p}$ ), and he was 5,800 grams 5 months after delivery. At the time of referral, his weight was 5,900 gr $\left(<3^{\text {rd }} \mathrm{p}\right)$, height was $64 \mathrm{~cm}\left(3^{\text {rd }}\right.$ $-10^{\text {th }} \mathrm{p}$ ), and head circumference was $42 \mathrm{~cm}$ $\left(10^{\text {th }} \mathrm{p}\right)$. Complete blood count, renal function tests, liver enzymes and liver function tests, electrolytes and blood gasses of the patient were normal. Fecal occult blood, fecal adenovirus, rotavirus, parasite and reducing substance test results were negative. Fecal fat and fat globules were positive, and stool steatocrit was $40 \%$ (normally $<10 \%$ ). His physical examination was noncontributory. CF was considered in the patient. Samples were sent for mutation analysis since the sweat chloride value was $94 \mathrm{mEq} / \mathrm{L}$ (normal value $<50 \mathrm{mEq} / \mathrm{L}$ ) in the sweat test. Pancreatic enzyme replacement and multivitamin supplementation was initiated.

Fifteen days following the initial admission, the patient was presented to the emergency department with restlessness and moaning. The general status of the patient was poor, tachypneic and tachycardic. Physical examination revealed a palpable liver $4 \mathrm{~cm}$ below the costal margin. Laboraty analysis revealed: hemoglobin $9.7 \mathrm{gr} / \mathrm{dl}$, white blood cell count $4,600 / \mathrm{mm}^{3}$ (absolute neutrophil count $900 / \mathrm{mm}^{3}$ ), and the platelet count $268,000 / \mathrm{mm}^{3}$; AST 382 $\mathrm{IU} / \mathrm{L}$, ALT $167 \mathrm{IU} / \mathrm{L}$, uric acid $11.2 \mathrm{mg} / \mathrm{dl}$, blood glucose $14 \mathrm{mg} / \mathrm{dl}$, phosphorus $11.2 \mathrm{mg} /$ $\mathrm{dl}$, ammonia $136 \mathrm{mmol} / \mathrm{L}$, lactate $106 \mathrm{mg} /$ $\mathrm{dl}$, and $\mathrm{CK} 505 \mathrm{U} / \mathrm{L}$ as positive biochemical findings, and $\mathrm{pH} 6.92, \mathrm{pCO}_{2} 43.3 \mathrm{~mm} \mathrm{Hg}$, $\mathrm{HCO}_{3} 7.4 \mathrm{mmol} / \mathrm{L}, \mathrm{pO}_{2} 79.6 \mathrm{~mm} \mathrm{Hg}, \mathrm{BE}$ $-21.4 \mathrm{mmol} / \mathrm{L}$ and oxygen saturation $86 \%$ as blood gas parameters. As cardiomegaly was detected by the telecardiography, supportive care was provided in the intensive care unit (Fig. 1). Despite the intensive treatments, the patient died three hours after admission. Postmortem liver biopsy was taken with the consent of the family.

In the urine organic acid analysis during follow-up, increased levels of lactic acid and dicarboxylic acid (adipic, suberic and sebacic acid) were found. In the tandem MS acylcarnitine profile, C14: 1, C14: 2, C10, C10: 1 and C8 levels were high and C5DC level was at the upper limit of normal. Additionally, a prominent peak was observed consistent with increased ketone bodies in the blood. In the amino acid profile, phenylalanine and alanine levels were high. In the post-mortem liver biopsy, approximately $5 \%$ of macrovesicular steatosis was detected.

In light of these findings, possible metabolic diseases were again discussed with the family. We learned that some close relatives in the family had died in their early childhood. The pedigree was obtained (Fig. 2). The family tree of the mother showed that her mother gave seven live births in eight pregnancies (she had one abortion). There were two abortions and six early deaths in the children of the siblings of the mother. Of the causes of death, one was suspected sepsis (boy), two were cardiomyopathy (boy), two were prematurity (girl), and one was chronic diarrhea (girl). Additionally, a nephew of the mother was diagnosed with BS in Germany. When further investigations were carried out for suspected $\mathrm{BS}$, clinical findings were found to be consistent with the disease. After having contacted the medical center in Germany, the mutation in their patient was learned to be the C51.G> C (p.Trp17X) mutation in the TAZ gene. Thus, we planned to investigate this mutation in our patient by communicating with a center capable of analyzing the mutation. 


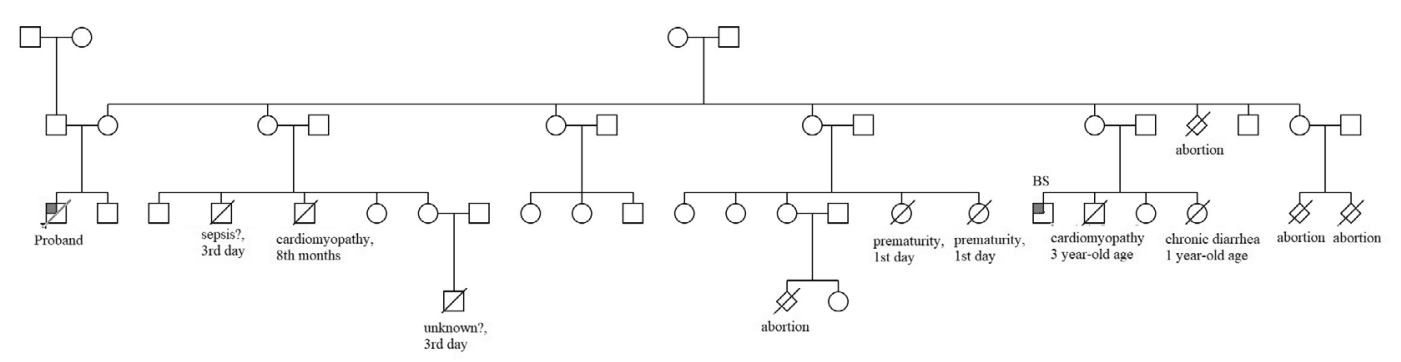

Fig. 2. Pedigree of the family

The results of CF gene analysis initiated during the follow-up were obtained. Three mutations were detected as compound heterozygous mutations: "[V754M / c.1408 G> A (p.Val470Met), single nucleotide polymorphism / c.4389 G>A, single nucleotide polymorphism]". Although the facts that the patient had steatorrhea, sweat test positivity, heterozygous mutation, two single gene polymorphisms and death of close relatives due to chronic diarrhea pointed out CF, it could not account for all symptoms of the patient. Further analysis revealed that the patient had exon 1 mutation c51.G> C (p.Trp17X); $T A Z$ gene mutation was also detected in our patient as previously described in his cousin. The genetic analysis carried out during the follow-up revealed that his mother was a BS carrier and his brother was healthy. The family was provided with genetic counseling and informed that prenatal diagnosis would be offered in future pregnancies if necessary. A prenatal diagnosis was attempted after three years, nonetheless, a center fully equipped for such an analysis was not found in our country. Fortunately, the postnatal genetic testing result was negative for BS

\section{Discussion}

In the present article, a six-month-old male patient with BS and CF was presented. Our patient had growth retardation, chronic diarrhea, cardiomegaly, neutropenia, hypoglycemia, myopathy, lactic acidosis and a family history consistent with BS. The metabolic analysis also provided data that could be compatible with BS. In addition to these findings, dysmorphic findings, recurrent septicemia and oral infections, mental and motor retardation, exercise intolerance, osteopenia, nutritional problems, ventricular arrhythmia, prolonged QT interval and left ventricular noncompaction can also be observed in BS. Furthermore, stillbirths, abortions, and hydrops fetalis might occur. ${ }^{2} \mathrm{CL}$ level screening can be one of the diagnostic criteria; however, accurate diagnosis is based on the detection of genetic mutations in patients with clinical findings compatible with the disease. Additionally; our patient had steatorrhea and sweat test positivity and mutations compatible with $\mathrm{CF}$.

In BS, the genetic inheritance has an X-linked recessive pattern. Accordingly, daughters of a carrier mother have a $50 \%$ risk of being a carrier and sons have a $50 \%$ risk of having BS. If the father has the disorder, the daughters will be born as carriers, and they will be healthy. Taking these facts into account, the deaths of nephews of the mother and abortion of her sister might have been related to BS. Additionally, the death of a brother of the mother due to abortion also seems to be related to BS. It has been shown that people with the same mutation might have different presentations. Whereas a mutation caused severe congestive heart failure necessitating heart transplant in short-term in one patient, the same mutation resulted in mild myopathy that could be diagnosed at the age of 43 years in another patient. ${ }^{4}$

In addition to clinical findings of BS, a decrease in CL level and an increase in monolysocardiolipin/CL ratio, which are used as screening tests, are of great importance for the diagnosis. Further diagnostic investigation is considered as a 5-20 fold increase of 3-MGA 
level in urine organic acid analysis and a genetic mutation in the TAZ gene. 3-methylglutaconic aciduria is also increased in mitochondrial diseases other than BS; however, this increase is not as much as the one observed in BS (2-3 fold). ${ }^{5}$ Moreover, as seen in our case, dicarboxylic aciduria, which suggests long chain fatty acid oxidation deficiencies in samples collected following long-term fasting or during the hypoglycemic crisis in the infantile period, and lactic aciduria in cardiac decompensation can be observed 6 .

It was thought that the coexistence of $\mathrm{CF}$ and BS in our case could be a coincidence. Although the coexistence of CS with some syndromes such as Silver-Russell syndrome, EhlersDanlos syndrome and Gilbert's syndrome and metabolic diseases such as phenylketonuria was previously described, the one with BS has not been reported in the literature until now. ${ }^{7-10}$ Both diseases have common clinical signs of chronic diarrhea, liver steatosis, osteopenia and growth retardation. However, the pathogenesis, epidemiology and genetic inheritance pattern of the diseases are completely different.

Detection of etiology in sudden unexpected infant deaths is often difficult, yet metabolic diseases such as fatty acid oxidation defects (3-6\%) and ketogenic defects, urea cycle disorders, organic acidemia, mitochondrial respiratory chain defects and carbohydrate metabolism disorders are among important causes $^{11}$. Hence, it is recommended to take liver, muscle and skin biopsies together with patient's whole blood, urine and CSF samples for diagnosis in the postmortem period. For the collection of all these samples, the consent of the family is required. In addition, the knowledge and experience of the clinician are important. Clinicians are generally reluctant to make postmortem diagnosis. In fact, several clues would have been collected for the diagnosis if the pedigree was obtained during the initial admission of the patient; however, the pedigree analysis is not performed in most patients due to the large volume of patients treated at outpatient clinics each day and the underestimation of the issue. In addition, taking the medical history of parents and brother could only have been considered sufficient due to the presence of findings compatible with CF during the initial presentation.
In conclusion, we have presented the coexistence of BS, a rare metabolic disease of childhood, with $\mathrm{CF}$, the most common genetic disease of Caucasians for the first time. We would like to emphasize the importance of extensive pedigree analysis which is difficult to perform in an outpatient clinic as well as the value of the collection of blood, urine and tissue specimens for postmortem diagnosis in cases with suspicion of metabolic disease.

\section{REFERENCES}

1. Barth PG, Scholte HR, Berden JA, et al. An $\mathrm{X}$-linked mitochondrial disease affecting cardiac muscle, skeletal muscle and neutrophil leucocytes. J Neurol Sci 1983; 62: 327-355.

2. Saric A, Andreau K, Armand AS, et al. Barth Syndrome: From mitochondrial dysfunctions associated with aberrant production of reactive oxygen species to pluripotent stem cell studies. Front Genet 2016; 20: 359. DOI: 10.3389/ fgene.2015.00359.

3. Farrell PM, White TB, Ren CL, et al. Diagnosis of cystic fibrosis: Consensus Guidelines from the Cystic Fibrosis Foundation. J Pediatr 2017; 18: S4-15.

4. Ronvelia D, Greenwood J, Platt J, Hakim S, Zaragoza, MV. Intrafamilial variability for novel TAZ gene mutation: Barth syndrome with dilated cardiomyopathy and heart failure in an infant and left ventricular noncompaction in his great-uncle. Mol Genet Metab 2012; 107: 428-432.

5. Vernon HJ, Sandlers Y, McClellan R, Kelley RI. Clinical laboratory studies in Barth syndrome. Mol Genet Metab 2014; 112: 143-147.

6. Waggoner DJ, Brown RL, Hedrick J. Hypoglycemia and dicarboxylic aciduria as the presenting biochemical findings in a patient with Barth syndrome. Am JHum Genet2001: 69: 492

7. Sonnappa S, Prescott K, Adler B, Dinwiddie $\mathrm{R}$ Wallis C. Cystic fibrosis and Russell-Silver syndrome in a child with maternal isodisomy of chromosome 7. Pediatr Pulmonol 2005; 40: 166-168.

8. Jarisch A, Giunta C, Zielen S, Konig R, Steinmann B. Sibs affected with both Ehlers-Danlos syndrome type IV and cystic fibrosis. Am J Med Genet 1998; 78: 455-460.

9. Wasmuth HE, Keppeler H, Herrmann U, SchirinSokhan R, Barker M, Lammert F. Coinheritance of Gilbert syndrome-associated UGT1A1 mutation increases gallstone risk in cystic fibrosis. Hepatology 2006; 43: 738-741. 
10. Kalkanoğlu HS, Anadol D, Yilmaz E, Coşkun T. Phenylketonuria and cystic fibrosis in the same patient. Pediatr Int 2000; 42: 92-93.
11. Olpin SE. The metabolic investigation of sudden infant death. Ann Clin Biochem 2004; 41: 282293. 